\title{
Nonparametric test for cointegration rank using Cholesky factor bootstrap
}

\author{
Jin Lee ${ }^{1, a}$ \\ ${ }^{a}$ Department of Economics, Ewha Womans University, Korea
}

\begin{abstract}
It is a long-standing issue to correctly determine the number of long-run relationships among time series processes. We revisit nonparametric test for cointegration rank and propose bootstrap refinements. Consistent with model-free nature of the tests, we make use of Cholesky factor bootstrap methods, which require weak conditions for data generating processes. Simulation studies show that the original Breitung's test have difficulty in obtaining the correct size due to dependence in cointegrated errors. Our proposed bootstrapped tests considerably mitigate size distortions and represent a complementary approach to other bootstrap refinements, including sieve methods.
\end{abstract}

Keywords: cointegration, long-run relationship, nonparametrics, Cholesky factor bootstrap, size refinements

\section{Introduction}

It has been a long-standing issue in the context of time series econometrics to determine the number of long-run equilibrium relationships among multivariate time series processes. The concept of cointegration is defined as existence of stationary linear combinations of nonstationary time series processes. In order to test for the number of cointegrations, most existing testing procedures typically rely on linear models that include vector autoregression (VAR) or error correction models. A popular test is Johansen (1995) likelihood-based test, which depend on VAR models. We also note recent approaches to present bootstrap refinements of Johansen's cointegration tests in Cavaliere et al. (2012) and Swensen (2006). Bootstrapped Johansen test also depend on a linear VAR model. The use of VAR-type models to derive the tests have a good advantage including tractability.

Our paper revisits Breitung (2002) nonparametric cointegration tests, which is the variance ratio tests. Thus, Breitung's test has a model-free statistic without assuming linear models, thus it is unaffected by selection of VAR lags or a specification of short-run dynamics. Regardless of such merit, we note that a finite sample performance of the tests are possibly questionable. The test has asymptotic limit distributions consisting of functionals of Brownian motions, which could be vulnerable to size distortions. It is expected, in the light of unit root and cointegrations contexts, that type I errors of the asymptotic tests increase as cointegrated errors become more serially correlated. Therefore, we employ appropriate bootstrap methods to Breitung's test as well as try to achieve possible size refinements. In particular, we consider Cholesky factor bootstrap developed in Diebold et al.

\footnotetext{
${ }^{1}$ Department of Economics, Ewha Womans University, 52 Ewhayeodae-gil, Seodaemun-gu, Seoul 03760, Korea.

E-mail: leejin@ewha.ac.kr
}

Published 30 November 2016 / journal homepage: http://csam.or.kr

(c) 2016 The Korean Statistical Society, and Korean International Statistical Society. All rights reserved. 
(1998). Recent research on cointegration mostly utilize sieve bootstraps for finite sample improvement (Berkowitz and Kilian, 2000; Bühlmann, 1997; Chang et al., 2006; Palm et al., 2010). However, unlike sieve bootstraps, Cholesky factor bootstraps do not require linear structures for underlying data generating processes, thus fit in nonparametric nature of the original Breitung's tests.

Our work summarizes Breitung's tests for cointegration ranks and proposes bootstrapped version in Section 2, and present some Monte Carlo simulation studies in Section 3. Section 5 concludes this study.

\section{Bootstrapping nonparametric test for cointegration}

\subsection{Model}

We first summarizes Breitung (2002) variance ratio test. Consider $n$-dimensional multivariate time series $z_{t}$,

$$
z_{t}=\delta^{\prime} D_{t}+z_{t-1}+u_{t},
$$

where $\delta$ is $m \times 1$ vector (the superscript' denotes the transpose) and the deterministic trends $D_{t}$ are specified as $D_{t}=1$, when $m=1$, or $D_{t}=(1, t)^{\prime}$, when $m=2$.

Decompose $z_{t}$ process into stochastic common trends and transitory (stationary) components (known as Stock and Watson's common trend representation in economics context). Write,

$$
\left.Q^{\prime}\left[z_{t}-E\left(z_{t}\right)\right]=\left(\alpha^{\prime}\left[z_{t}-E\left(z_{t}\right)\right], \beta^{\prime} z_{t}-E\left(z_{t}\right)\right]\right)^{\prime}=\left(w_{t}, v_{t}\right)^{\prime},
$$

where $Q \in R^{n \times n}$, for $\alpha \in R^{n \times q}$ and $\beta \in R^{n \times r}$ with $q+r=n$, respectively, and $E\left(z_{t}\right)=\delta_{t}^{\prime}$.

The number of stochastic trends equal to $q$, and the number of cointegrations is $r$, where the linear combination $\beta^{\prime}\left[z_{t}-E\left(z_{t}\right)\right]$ represents cointegrating relations. It is noted that it is not necessary to specify short-run dynamics for cointegrated errors $v_{t}$.

Let $\hat{z}_{t}=z_{t}-\hat{\delta}^{\prime} D_{t}$, and define the matrices,

$$
A_{T}=\sum_{t=1}^{T} \hat{z}_{t} \hat{z}_{t}^{\prime}, \quad B_{T}=\sum_{t=1}^{T} \hat{S}_{t} \hat{S}_{t}^{\prime},
$$

where $\hat{S}_{t}$ is the partial sums, $\hat{S}_{t}=\sum_{j=1}^{t} \hat{z}_{j}$. Consider eigenvalues $(\lambda)$ of the matrix $A_{T} B_{T}^{-1}$, and put the ordered eigenvalues $\lambda_{1} \leq \lambda_{2} \leq \cdots \leq \lambda_{T}$. The original idea of Breitung (2002) test states that if the eigenvector belongs to the space spanned by the columns of $\alpha$, then $T^{2} \lambda_{j}=O_{p}(1)$. Otherwise, if eigenvectors belong to the space spanned by cointegrating vectors, then $T^{2} \lambda_{j} \rightarrow \infty$, as $T \rightarrow \infty$.

The test statistic takes the following form (Breitung, 2002, Section 5),

$$
V_{q}=T^{2} \sum_{j=1}^{q} \lambda_{j}
$$

Under the null hypothesis that there are exactly $q$ stochastic trends (for $0<q \leq n$ ),

$$
V_{q} \stackrel{d}{\rightarrow} \operatorname{tr} \frac{\left(\int_{0}^{r} W(r) W(r)\right)}{\left(\int_{0}^{r} U(r) U(r)\right)}, \text { for } r(0,1),
$$

where $W(r)$ is $q$-dimensional detrended Brownian motion and $U(r)=\int_{0}^{r} W(s) d s$. The symbol $\stackrel{d}{\rightarrow}$ denotes the convergence in distribution and $\operatorname{tr}(A)$ means the trace of the matrix $A$. Under the alternative hypothesis that there are less than $q$ trends, then $V_{q}$-test diverges. 


\subsection{Bootstrap methods}

We now consider suitable bootstrap methods in order to mitigate possible size distortion of the asymptotic tests. Consistent with nonparametric nature of the test, we employ Cholesky factor bootstrap, instead of sieve bootstraps (Diebold et al., 1998).

Our proposed bootstrap algorithms are given as:

(a) Obtain the detrended series $\hat{z}_{t}=z_{t}-\hat{\delta}^{\prime} D_{t}$ and let $\Delta z_{t}=z_{t}-z_{t-1}$.

(b) Let $\hat{\Sigma}$ be the heteroskedasticity and autocorrelation consistent (HAC) covariance matrix estimator of variance-covariance of $\Delta z_{t}$,

$$
\hat{\Sigma}=\sum_{j=1-T}^{T-1} k\left(\frac{j}{M}\right) \hat{\Gamma}(j)=\hat{\Gamma}(0)+2 \sum_{j=1}^{T-1} k\left(\frac{j}{M}\right)\left(\hat{\Gamma}(j)+\hat{\Gamma}(j)^{\prime}\right),
$$

where

$$
\hat{\Gamma}(j)= \begin{cases}T^{-1} \sum_{t=j+1}^{T} \Delta z_{t} \Delta z_{t-j}, & j \geq 0, \\ T^{-1} \sum_{t=j+1}^{T} \Delta z_{t+j} \Delta z_{t}, & j<0 .\end{cases}
$$

The $k()$ is a kernel function. The bandwidth $M$ is selected by a data-dependent method. For example, the nonparametric plug-in method of Newey and West (1994) is used in our work. The optimal bandwidth in the case of using Bartlett kernel is known as,

$$
M=\hat{\gamma} T^{\frac{1}{3}} \quad \text { and } \quad \hat{\gamma}=1.1447\left(\frac{\hat{f}^{(1)}(0)}{\hat{f}(0)}\right)^{\frac{2}{3}},
$$

where $\hat{f}^{(1)}(0)$ and $\hat{f}(0)$ equal to the first spectral derivative and the spectral density estimator at the zero frequency, respectively, given by

$$
\hat{f}^{(1)}(0)=2 \sum_{j=1}^{m} j \times \Gamma(j), \quad \hat{f}(0)=\Gamma(0)+2 \sum_{t=1}^{m} \Gamma(j),
$$

and $m$ is the lag truncation number (Newey and West, 1994, Table II).

(c) Write $\hat{\Sigma}=\hat{P} \hat{P}$, where $\hat{P}$ is a lower triangular matrix. Denote $\hat{P}$ as a Cholesky factor. By construction, we have

$$
\Delta \hat{z}_{t}=\hat{P} e_{t},
$$

where $e_{t}$ is i.i.d. with $E\left(e_{t} e_{t}\right)=I_{n}$ (equivalently, $\hat{P}^{-1} \Delta z_{t}=e_{t}$ ); Thus, $\hat{P}$ plays a role of a sieve in sieve bootstraps.

(d) Let $\tilde{e}_{t}=e_{t}-\bar{e}_{t}$, where $\bar{e}_{t}$ is the sample mean of $\hat{P}^{-1} \Delta z_{t}$. Resample $\tilde{e}_{t}$ from recentered $\hat{P}^{-1} \Delta z_{t}$ and denote it as $\tilde{e}_{t}^{*}$. 
(e) Get the bootstrap samples from $\Delta \hat{z}_{t}^{*}=\hat{P} \tilde{e}_{t}^{*}$, and $\hat{z}_{t}^{*}=\Delta \hat{z}_{t}^{*}+\hat{z}_{t-1}^{*}$. Construct a bootstrapped test statistic $V_{q}^{*}$.

(f) Repeat the above steps many times and obtain the $100 \times \alpha \%$ critical values, denoted as $C V_{\alpha}^{*}$, from the empirical distribution of $V_{q}^{*}$.

Finally, we reject the null hypothesis at the $100 \times \alpha \%$ significance level if

$$
V_{q}>C V_{\alpha}^{*}
$$

Some remarks can be made for the above algorithms. A main difference between sieve bootstrap and Cholesky factor bootstrap lies in steps (c) and (d). In the case of sieve bootstraps, lag order selection in autoregressive models becomes an issue. However, Cholesky factor bootstraps create an issue on the choice of the bandwidth in the above covariance matrix estimation. Therefore, lag orders and bandwidths play similar roles in both bootstrap procedures.

We note that the use of Cholesky factor bootstraps can be a complementary approach to sieve bootstrap methods (Bühlmann, 1997; Chang et al., 2006). To apply sieve methods, linear structures of the data generating processes need to be assumed, which is not required for the use of Cholesky factor bootstraps.

\section{Simulations}

We now analyze finite sample performance of Breitung's test and the bootstrapped counterpart. The simulation design follows from bivariate linear models in Toda (1995). Through simulations, we investigate whether the proposed bootstrap methods also work in widely used linear models. Consider a cointegrated model that consists of bivariate time series processes,

$$
\begin{aligned}
& x_{t}=\delta_{1}+\phi x_{t-1}+u_{1 t}, \\
& y_{t}=\delta_{2}+y_{t-1}+u_{2 t},
\end{aligned}
$$

where errors follow AR(1) processes,

$$
u_{1 t}=\theta u_{1 t-1}+e_{1 t}, \quad u_{2 t}=\theta u_{2 t-1}+e_{2 t},
$$

and $e_{t}=\left(e_{1 t}, e_{2 t}\right)$ is iid $N(0, \omega)$ with $\omega_{[11]}=\omega_{[22]}=1$, and $\omega_{[12]}=\gamma$.

Sample sizes $T=100$ and $T=200$ are considered, as consistent with most economic time series data. The number of replications equals to 5,000. We set $\delta_{1}=\delta_{2}=0$ in this simulation. For serially correlated errors, a range of the $\mathrm{AR}(1)$ coefficients are included, i.e., $\theta$ from -0.4 to 0.4 . Note that Toda (1995) includes iid errors in the simulation, and Breitung (2002) only investigates MA(1) error (moving average error with the order 1). Therefore, out setup allows stronger dependence in cointegrated errors. To save the space, we set $\gamma=0$.

The cointegration ranks refer to the value of $\phi$. we include two cases to figure out the size performance. First, we consider (i) $\phi=1$, then there exist two stochastic trends (no cointegration exists). Second, we include the case that $\phi=0.8$, where one stochastic trend (one cointegration) is present. This follows from the setup in Toda (1995).

Table 1 and Table 2 present the size performance at the 5\% level. The finding is summarized as follows. (i) Under the null of two stochastic trends (no cointegration), size of the tests sensitively depend on the correlation of error terms. For instance, the asymptotic $V$ test suffers more severe 
Table 1: Size performance of the variance ratio tests at the $5 \%$ level: $T=100$

\begin{tabular}{cccccc}
\hline \hline Demeaned case & \multicolumn{5}{c}{ AR $\theta$} \\
test & -0.4 & -0.2 & 0 & 0.2 & 0.4 \\
\cline { 2 - 6 }$(q=2) V$ & 7.38 & 5.76 & 4.58 & 3.68 & 3.00 \\
$V^{*}$ & 6.12 & 5.28 & 4.70 & 4.14 & 3.58 \\
\hline$(q=1) V$ & 2.44 & 2.04 & 1.60 & 1.24 & 0.96 \\
$V^{*}$ & 7.22 & 5.76 & 5.02 & 4.62 & 4.38 \\
\hline \hline
\end{tabular}

The values are rejection rates as the percentage values.

$q=$ number of stochastic trends; $V=$ asymptotic test; $V^{*}=$ bootstrap test from 1,000 bootstrap iterations of Cholesky factor bootstraps.

Table 2: Size performance of the variance ratio tests at the 5\% level: $T=200$

\begin{tabular}{cccccc}
\hline \hline Demeaned case & \multicolumn{5}{c}{ AR $\theta$} \\
test & -0.4 & -0.2 & 0 & 0.2 & 0.4 \\
\hline$(q=2) V$ & 6.58 & 5.64 & 5.04 & 4.56 & 3.90 \\
$V^{*}$ & 5.98 & 5.32 & 5.00 & 4.70 & 4.40 \\
\hline$(q=1) V$ & 2.36 & 2.04 & 1.74 & 1.54 & 1.30 \\
$V^{*}$ & 7.64 & 5.94 & 5.20 & 4.56 & 4.14 \\
\hline \hline
\end{tabular}

The values are rejection rates as the percentage values.

$q=$ number of stochastic trends; $V=$ asymptotic test; $V^{*}=$ bootstrap test from 1,000 bootstrap iterations of Cholesky factor bootstraps.

under-rejections when the correlation increases. The bootstrapped $V^{*}$ test rather mitigates the underrejections to some degree. However, we noted that when the errors are strongly correlated such as when $\theta=0.4$, the performance of the bootstrapped test is also unsatisfactory. (ii) Size refinements are more pronounced when one stochastic trend (one cointegration) is present. The asymptotic test fails to obtain correct size, where the rejection probability is only about $2 \%$ at the $5 \%$ level. However, the bootstrapped tests generates the size around the 5\% level, except for the large negative autocorrelation. (iii) We investigate the behavior of the tests for larger sample size of $T=200$. The unbootstrapped tests severely under-reject with no exceptions, whereas the bootstrapped versions behave reasonably, with the rejection probability close to the $5 \%$.

We also note that there is a room to improve the finite sample performance of the proposed tests by adjusting the bandwidth selections. First, unlike mean squared error (MSE)-optimal bandwidths as in Newey and West (1994), one may consider alternative bandwidth selections. This is a nontrivial task, though. Second, we restrict our attention to the simple VAR model in the simulation design. Therefore, we only conclude that Cholesky factor bootstrap methods work well in linear models despite a design that is applicable for nonlinear models.

\section{Application}

A brief empirical application is presented in this section by applying the proposed methods to real data. In doing so, we consider a possible cointegrating relationship between short-term and longterm interest rates of Korea. It is a crucial task to investigate if short-term interest rate and long-term interest rate tend to move together in the long-run. The 3 months $\mathrm{CD}$ rate is used for the short rate versus the 10-year government bond rate that is used for long rate. The data ranges from the first quarter of 2001 to the first quarter of 2015, which are obtained from the dataset of Federal Reserve Bank (FRB), St. Louis, USA. Both series are logged in the analysis.

We use both an asymptotic test and bootstrap test with Cholesky factor bootstrap. The null hypothesis states that there is no cointegration between the two interest rates. To implement the bootstrap 
tests, we run 1,000 bootstrap iteration to obtain the bootstrap critical values. The test statistic is computed as 269.46 . The asymptotic critical values equal to $67.89,95.6$ and 185 , at the $10 \%$, 5\%, and $1 \%$, respectively. Therefore, the test strongly rejects the nul of no cointegration even at the $1 \%$ significance level. However, bootstrap critical values are computed as 157.16, 203.38 and 315.31 at the 10\%, 5\%, and $1 \%$ level, respectively. It follows that the tests reject the null hypothesis both at the $10 \%$ and $5 \%$ levels, but not at the $1 \%$ level. We also conducted tests with unlogged interest rates and obtain similar results. In sum, it is found that the use of bootstrap methods lead to weaker rejection of cointegration between the two interest rates. Data and gauss codes are available upon request.

\section{Conclusion}

We revisit nonparametric test for cointegration rank by Breitung (2002) and propose bootstrap refinements by making use of Cholesky factor bootstrap methods. The original Breitung's test is based on asymptotic inferences that have difficulty in obtaining correct size due to serial correlation in cointegrated errors. We employ nonparametric Cholesky factor bootstraps in due to the nonparametric nature of the testing procedure. Thus, it is comparable to sieve bootstrap methods that require linear structure of the underlying time series processes. Simulation studies show that the proposed bootstrapped tests mitigate size distortions and yield a more reasonable size performance than unbootstrapped version.

\section{Acknowledgements}

The author is grateful to Joon Park for helpful comments and seminar participants at the Korean Econometric Society Conference in 2015.

\section{References}

Berkowitz J and Kilian L (2000). Recent developments in bootstrapping time series, Econometric Reviews, 19, 1-48.

Breitung J (2002). Nonparametric tests for unit roots and cointegration, Journal of Econometrics, 108, 343-363.

Bühlmann P (1997). Sieve bootstrap for time series, Bernoulli, 3, 123-148.

Cavaliere G, Rahbek A, and Robert Taylor AM (2012). Bootstrap determination of the co-integration rank in vector autoregressive models, Econometrica, 80, 1721-1740.

Chang Y, Park JY, and Song K (2006). Bootstrapping cointegrating regression, Journal of Econometrics, 133, 703-739.

Diebold FX, Ohanian LE, and Berkowitz J (1998). Dynamic equilibrium economies: a framework for comparing models and data, Review of Economic Studies, 65, 433-451.

Johansen S (1995). Likelihood-Based Inference in Cointegrated Vector Autoregressive Models, Oxford University Press, Oxford.

Newey WK and West KD (1994). Automatic lag selection in covariance matrix estimation, The Review of Economic Studies, 61, 631-653.

Palm FC, Smeekes S, and Urbain JP (2010). A sieve bootstrap test for cointegration in a conditional error correction model, Econometric Theory, 26, 647-681.

Swensen AR (2006). Bootstrap algorithms for testing and determining the cointegrating rank in VAR models, Econometrica, 74, 1699-1714.

Toda HY (1995). Finite sample performance of likelihood ratio tests for cointegration ranks in vector autoregressions, Econometric Theory, 11, 1015-1032. 